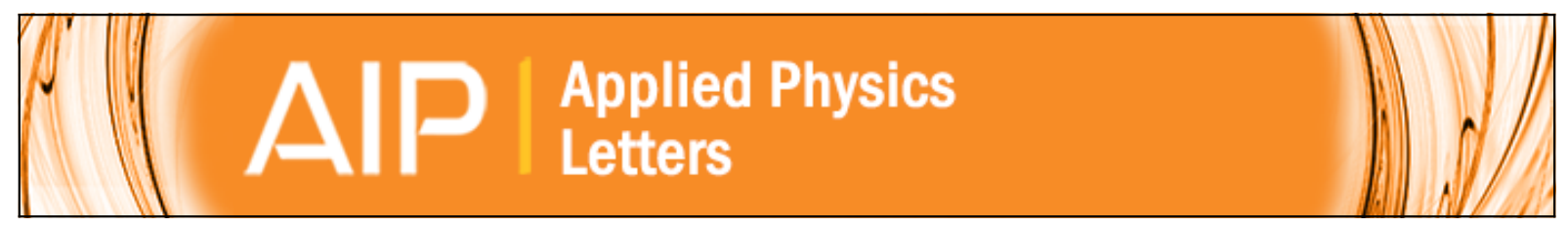

\title{
Atomic force microscope cantilevers for combined thermomechanical data writing and reading
}

William P. King, Thomas W. Kenny, Kenneth E. Goodson, Graham Cross, Michel Despont, Urs Dürig, Hugo Rothuizen, Gerd K. Binnig, and Peter Vettiger

Citation: Applied Physics Letters 78, 1300 (2001); doi: 10.1063/1.1351846

View online: http://dx.doi.org/10.1063/1.1351846

View Table of Contents: http://scitation.aip.org/content/aip/journal/apl/78/9?ver=pdfcov

Published by the AIP Publishing

\section{Articles you may be interested in}

Direct simulation Monte Carlo on thermal distribution of rarefied gas under heated atomic force microscope nanoprobe

J. Appl. Phys. 105, 013508 (2009); 10.1063/1.3054549

Writing and erasing efficiency analysis on optical-storage media using scanning surface potential microscopy J. Vac. Sci. Technol. A 24, 2003 (2006); 10.1116/1.2345641

The impact of subcontinuum gas conduction on topography measurement sensitivity using heated atomic force microscope cantilevers

Phys. Fluids 17, 100615 (2005); 10.1063/1.1932313

Inverse problem of scanning force microscope force measurements

J. Appl. Phys. 94, 3563 (2003); 10.1063/1.1592871

Ultrahigh-density atomic force microscopy data storage with erase capability

Appl. Phys. Lett. 74, 1329 (1999); 10.1063/1.123540

\section{A|P| Journal of}

Journal of Applied Physics is pleased to announce André Anders as its new Editor-in-Chief 


\title{
Atomic force microscope cantilevers for combined thermomechanical data writing and reading
}

\author{
William P. King, ${ }^{\text {a) }}$ Thomas W. Kenny, and Kenneth E. Goodson \\ Department of Mechanical Engineering, Stanford University, Stanford, California 94305-3030 \\ Graham Cross, Michel Despont, Urs Dürig, Hugo Rothuizen, Gerd K. Binnig, \\ and Peter Vettiger \\ IBM Research, Zurich Research Laboratory, 8803 Rüschlikon, Switzerland
}

(Received 13 October 2000; accepted for publication 3 January 2001)

\begin{abstract}
Heat conduction governs the ultimate writing and reading capabilities of a thermomechanical data storage device. This work investigates transient heat conduction in a resistively heated atomic force microscope cantilever through measurement and simulation of cantilever thermal and electrical behavior. The time required to heat a single cantilever to bit-writing temperature is near $1 \mu \mathrm{s}$ and the thermal data reading sensitivity $\Delta R / R$ is near $1 \times 10^{-4}$ per vertical $\mathrm{nm}$. Finite-difference thermal and electrical simulation results compare well with electrical measurements during writing and reading, indicating design tradeoffs in power requirements, data writing speed, and data reading sensitivity. We present a design for a proposed cantilever that is predicted to be faster and more sensitive than the present cantilever. (C) 2001 American Institute of Physics.
\end{abstract}

[DOI: $10.1063 / 1.1351846]$

In current magnetic data storage, the superparamagnetic effect limits magnetic domain stability and will ultimately limit room-temperature magnetic data density near 100 $\mathrm{Gb} / \mathrm{in}^{2}{ }^{1}{ }^{1}$ Several promising alternative data storage technologies that could surpass this limit involve the use of scanningprobe devices. ${ }^{2}$ Thermomechanical data storage is one such technology, ${ }^{3-5}$ demonstrating data density as high as 400 $\mathrm{Gb} / \mathrm{in}^{2}{ }^{5}$. In thermomechanical data storage, a resistively heated atomic force microscope (AFM) cantilever writes a data bit by scanning over a polymer substrate. Heat and force applied by the cantilever tip to the polymer cause it to soften and flow, writing a data bit, as shown in Fig. $1 .^{3-5}$ The same cantilever can be used to detect the presence of a previously written data bit. ${ }^{5}$ As the cantilever tip follows the contour of a data bit, the reduction in thermal impedance between the cantilever and the substrate causes a lower temperature rise in the heated cantilever, also shown in Fig. 1.

Previous research on thermomechanical data storage has focused on improving both data density and data rate. Silicon cantilevers with small solid-state resistive heaters near the cantilever tip allowed reduction of the cantilever heating time. ${ }^{4}$ Array operation of heater cantilevers allows higher data rates than a single cantilever configuration. We have recently fabricated a functional array of $32 \times 32$ heater cantilevers ${ }^{6,7}$ and report parallel writing and reading operation of this array in Ref. 7. Binnig et al. ${ }^{5}$ demonstrated a data density of $400 \mathrm{~Gb} / \mathrm{in}^{2}{ }^{2}$ by writing thermomechanical data bits of diameter $40 \mathrm{~nm}$ in a poly-methylmethacrylate (PMMA) layer of thickness $40 \mathrm{~nm}$. The very thin polymer layer confines the polymer melted region to dimensions on the order of the polymer thickness, and limits tip penetration and therefore data bit size. Recent research investigated heat

\footnotetext{
a) Also with IBM Research, Zurich Research Laboratory, Säumerstrasse 4/Postfach, 8803 Rüschlikon, Switzerland; electronic mail: wking@vk.stanford.edu
}

transfer during thermal data writing and reading. King and co-workers ${ }^{8}$ showed that the temperature of the tip-polymer interface is significantly higher than the temperature induced in the substrate due to the presence of the heater cantilever alone. These authors also showed that only a fraction of the total heat is transferred through the tip, and that much more of the heat passes across the cantilever-sample air gap, ${ }^{9}$ concluding that the presence of the heated tip permits data writing and that the presence of the heater cantilever permits data reading. Simultaneous improvement of cantilever writing and reading figures of merit is therefore possible, ${ }^{10}$ given
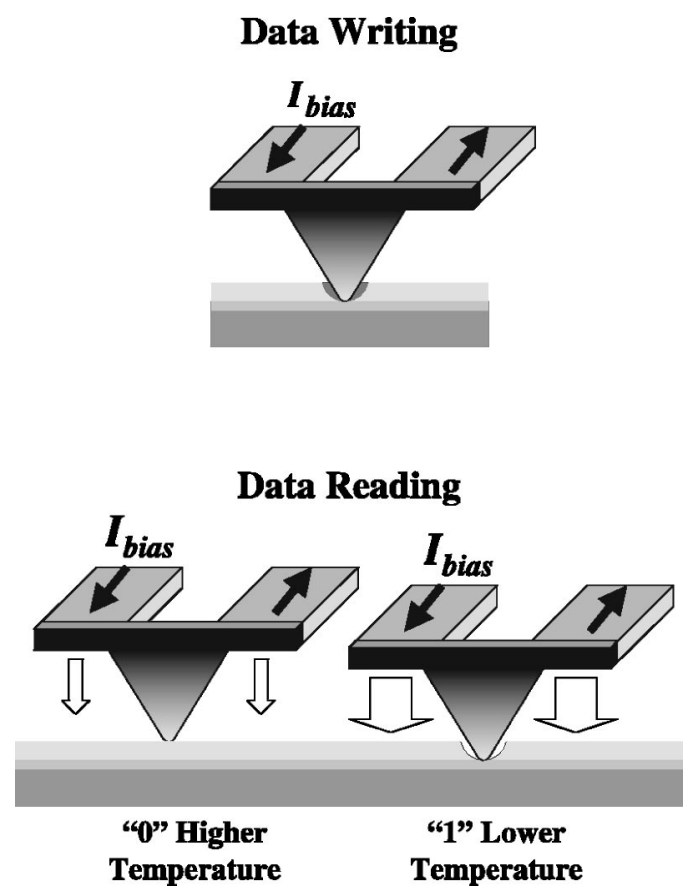

FIG. 1. Thermomechanical data bit writing and thermal data reading with a resistively heated AFM cantilever. 


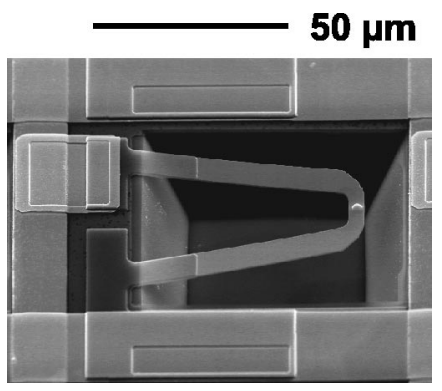

FIG. 2. Scanning electron microscopy image of the measurement cantilever.

detailed measurement and modeling of cantilever behavior.

This letter reports measurement and simulation of single-cantilever operation during data bit writing and thermal data reading. An electrical and thermal simulation predicts operation of a single cantilever identical in design to the array cantilever of Despont $e t a l .^{6}$ and is verified by electrical measurements. Figure 2 shows a scanning electron micrograph of the present cantilever. The simulation is then used to analyze a cantilever of a proposed new design. Table I lists the design attributes of both cantilevers. The proposed cantilever is designed with a smaller heater region to reduce the heating time ${ }^{5}$ and has a shorter tip to increase the data reading sensitivity. At $200 \mathrm{~nm}$ thickness, the present cantilever is the thinnest we have fabricated to date, for maximum thermal data reading sensitivity. ${ }^{9}$ The remaining cantilever dimensions are selected so as to achieve a 50\% improvement in the cantilever mechanical resonant frequency. The cantilever mechanical resonant frequency will approximately govern the shortest time between repeated bit writing and reading events (see, for example, Ref. 11).

The writing measurement is made by placing the cantilever in series with a sense resistor of $3.3 \mathrm{k} \Omega$, and square voltage pulses of duration 1-25 $\mu$ s and pulse amplitude of $1-15 \mathrm{~V}$ drive the circuit. The measurement of the voltage across the circuit and the sense resistor allows the calculation of cantilever resistance. Data reading sensitivity measurements are made by slowly moving the heated cantilever into contact with the polymer surface.

A finite-difference simulation calculates electrical heat generation in the cantilever and heat transfer along the cantilever and into the nearby air, with a spatial resolution of 50 $\mathrm{nm}$ and an explicit time advancement of $1 \mathrm{~ns}$. Circuit design models ${ }^{12}$ calculate the temperature-dependent intrinsic carrier generation and the temperature-dependent electrical resistivity of the doped silicon. The thermal conductivity of the highly doped silicon cantilever is assumed to be 50 $\mathrm{Wm}^{-1} \mathrm{~K}^{-1}$ at room temperature ${ }^{13}$ and vary as the inverse of absolute temperature. For each simulation time advance-

TABLE I. Design details for present and proposed cantilevers.

\begin{tabular}{lcc}
\hline \hline & Present cantilever & Proposed cantilever \\
\hline Heater area & $5 \mu \mathrm{m} \times 7 \mu \mathrm{m}$ & $2 \mu \mathrm{m} \times 5 \mu \mathrm{m}$ \\
Thickness & $200 \mathrm{~nm}$ & $200 \mathrm{~nm}$ \\
Tip height & $500 \mathrm{~nm}$ & $200 \mathrm{~nm}$ \\
Leg width & $10 \mu \mathrm{m}$ & $5 \mu \mathrm{m}$ \\
Leg length & $50 \mu \mathrm{m}$ & $20 \mu \mathrm{m}$ \\
Electrical resistance at $25^{\circ} \mathrm{C}$ & $2120 \Omega$ & $2012 \Omega$ \\
Resonant frequency indicated in the $220 \mathrm{kHz}$ Reuse of $330 \mathrm{kHz}$ is is s \\
\hline \hline
\end{tabular}

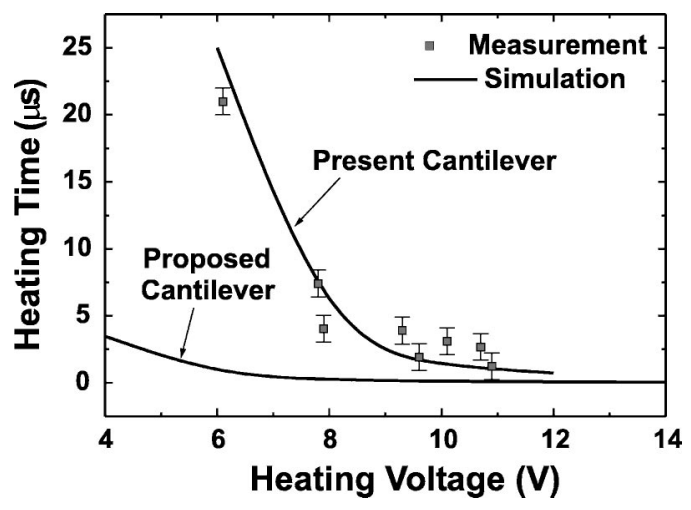

FIG. 3. Measurement and simulation of the time required to reach the bitwriting temperature as a function of voltage applied to the cantilever.

ment, the simulation calculates the total cantilever electrical resistance, which determines the cantilever current at fixed driving voltage. The cantilever current determines the heating power at each position along the length of the cantilever. The simulation agrees with analytical solutions to within $2 \%$, and further variation between measurement and simulation is due to noise and parasitic capacitance in the cantilever.

Figure 3 shows measurement and prediction of the time required for the cantilever to reach the writing temperature. The thermomechanical writing temperature is taken to be $350^{\circ} \mathrm{C}$, which is the measured writing temperature in thin PMMA. ${ }^{5,7}$ Predictions indicate that the lowest applied voltage at which the present cantilever can write data is near $6 \mathrm{~V}$.

Figure 4 shows measurement and prediction of the energy required for a cantilever to write a single data bit. The diffusion of heat is confined to a smaller region in the cantilever for shorter heating pulses of higher voltage, which corresponds to the lower energy operation. The design of a thermomechanical data storage system must balance the requirements of low power and voltage with the requirements of low energy consumption.

Figure 5 shows measurement and prediction of the thermal data reading sensitivity. Higher heating power corresponds to a greater change in the fraction of the heat that is transferred across the cantilever-substrate air gap for a given change in gap height. The cantilever temperature should not be high enough to deform the polymer during reading. The nonlinearity in the curve is due in part to the change in cantilever electrical resistance with temperature. Tips much

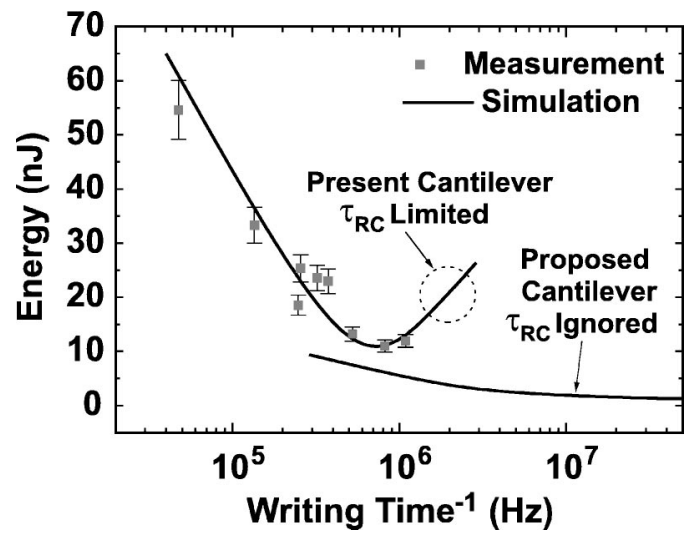

FIG. 4. Measurement and simulation of the energy required for a single cantilever to write a data bit as a function of heating time. 


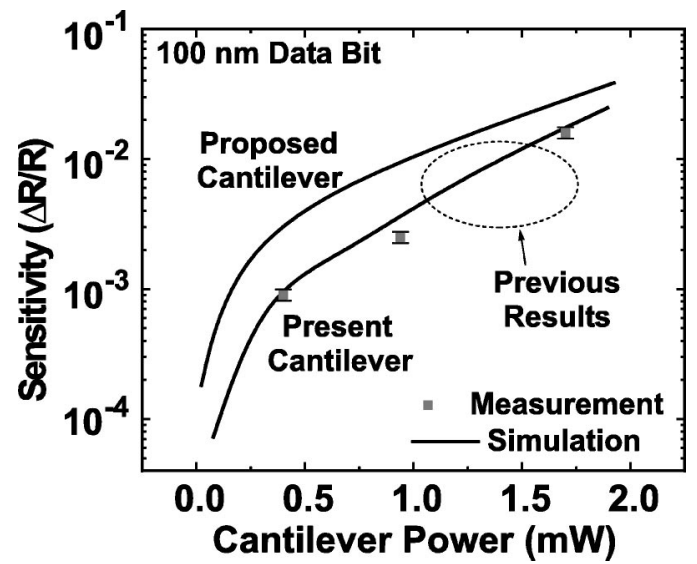

FIG. 5. Measurement and prediction of thermal data reading calculated for reading a data bit of depth $100 \mathrm{~nm}$. Previous data-reading sensitivity measurements are also indicated (Refs. 5 and 7).

shorter than $200 \mathrm{~nm}$ will probably not improve reading sensitivity significantly as air rarefaction becomes important.

Predictions for the operation of the proposed cantilever indicate that this improved design offers important reductions of the heating time, power and energy requirements, and improves reading sensitivity. In particular, the lowest voltage at which a data bit could be written is nearly halved by the proposed cantilever, and the heating time is also reduced.

This work analyzes the impact of heat conduction in a resistively heated cantilever during thermomechanical data writing and reading and presents comprehensive thermal design of cantilevers for thermomechanical data storage. It is possible to improve at the same time both the heating characteristics that govern data bit writing and the sensitivity governing data bit reading. Although the proposed cantilever could be fabricated with no changes in the fabrication process, we envision forefront fabrication technology to realize the ultimate limits of speed, power, and sensitivity in thermomechanical data storage. The ultimate limits of thermomechanical data storage will further depend upon electronics integration and engineering of the polymer data layer.
The authors thank M. Asheghi, B. Chui, K. Kurabayashi, M. Lutwyche, J. Santiago, P. Seidler, and P. Sverdrup for valuable encouragement and discussions. We acknowledge the collaboration of U. Drechsler, G. Genolet, W. Häberle, R. Stutz, and R. Widmer of the IBM Zurich Micro/ NanoMechanics Group. W. P. K. appreciates the support of IBM Research and the IBM Graduate Research Fellowship Program. K. E. G. was supported by an ONR Young Investigator Award.

${ }^{1}$ E. Grochowski and R. F. Hoyt, IEEE Trans. Magn. 32, 1850 (1996); D. A. Thompson and J. S. Best, IBM J. Res. Dev. 44, 311 (2000).

${ }^{2}$ E. B. Cooper, S. R. Manalis, H. Fang, H. Dai, K. Matsumoto, S. C. Minnie, T. Hunt, and C. F. Quate, Appl. Phys. Lett. 75, 3566 (1999); H. J. Mamin, R. P. Ried, B. D. Terris, and D. Rugar, Proc. IEEE 87, 1014 (1999).

${ }^{3}$ H. J. Mamin, Appl. Phys. Lett. 69, 433 (1996).

${ }^{4}$ B. W. Chui, T. D. Stowe, T. W. Kenny, H. J. Mamin, B. D. Terris, and D. Rugar, Appl. Phys. Lett. 69, 2767 (1996); B. W. Chui, T. D. Stowe, Y. S. Ju, K. E. Goodson, T. W. Kenny, H. J. Mamin, B. D. Terris, R. P. Ried, and D. Rugar, J. Microelectromech. Syst. 7, 69 (1998).

${ }^{5}$ G. Binnig, M. Despont, U. Drechsler, W. Häberle, M. Lutwyche, P. Vettiger, H. J. Mamin, B. W. Chui, and T. W. Kenny, Appl. Phys. Lett. 74, 1329 (1999).

${ }^{6}$ M. Despont, J. Brugger, U. Drechsler, U. Dürig, W. Häberle, M. Lutwyche, H. Rothuizen, R. Stutz, R. Widmer, H. Rohrer, G. K. Binnig, and P. Vettiger, Sens. Actuators A 80, 100 (2000).

${ }^{7}$ M. I. Lutwyche, G. Cross, M. Despont, U. Drechsler, U. Dürig, W. Häberle, H. Rothuizen, R. Stutz, R. Widmer, G. K. Binnig, and P. Vettiger, Appl. Phys. Lett. 77, 3299 (2000); P. Vettiger, M. Despont, U. Drechsler, U. Dürig, W. Häberle, M. I. Lutwyche, H. Rothuizen, R. Stutz, R. Widmer, and G. Binnig, IBM J. Res. Dev. 44, 323 (2000).

${ }^{8}$ W. P. King, J. G. Santiago, T. W. Kenny, and K. E. Goodson, Proc. ASME MEMS 1, 583 (1999).

${ }^{9}$ M. Asheghi, W. P. King, B. W. Chui, T. W. Kenny, and K. E. Goodson, Proc. Transducers '99 Tech. Dig. 1, 1840 (1999).

${ }^{10}$ W. P. King, T. W. Kenny, K. E. Goodson, G. Cross, M. Despont, U. Dürig, M. I. Lutwyche, H. Rothuizen, G. K. Binnig, and P. Vettiger, Hilton Head 2000 Solid-State Sensors and Actuator Workshop Technical Digest (2000), pp. 1-5.

${ }^{11}$ R. P. Ried, J. Mamin, B. D. Terris, L. S. Fan, and D. Rugar, J. Microelectromech. Syst. 6, 294 (1997).

${ }^{12}$ S. M. Sze, Physics of Semiconductor Devices (Wiley, New York, 1981); S. Selberherr, Microelectron. Reliab. 24, 225 (1984).

${ }^{13}$ M. Asheghi, K. Kurabayashi, R. Kasnavi, and K. E. Goodson (unpublished). 